\title{
Homology Modelling of Lycopene Cleavage Oxygenase: The Key Enzyme of Bixin Production
}

\author{
Raghunath Satpathy*, Rajesh Ku.Guru, Rashmiranjan Behera and Aparajita Priyadarshini \\ Department of Biotechnology, MIRC LAB, MITS Engineering College, Rayagada, Odisha, India
}

\begin{abstract}
Bixin is a natural dye and a high commercial important compound, produced from Bixin synthetic pathway in case of Bixa orellana plant. The particular enzyme Lycopene cleavage Oxygenase catalyzes the first step of reaction pathways from Trans-lycopene to Bixin synthesis. The 3D structure of the enzyme was predicted by MODELLER program and the missing side chains were verified by SCRWL4 tool. Model validation was done by using the output of PROCHECK and DOPE score. The Ramachandran plot for the model was observed as 87.3 percentages of residues is in favourable regions that indicate the model is reliable. Molecular dynamics simulation of the model protein was performed in water 5 lakh times for 1000 Pico second and at 300K by GROMACS software in a high performance computing environment. The energy value and RMSD (Root men square deviation) fluctuation of Carbon alpha back bone of the model was computed that confirms the stability of the model protein.
\end{abstract}

Keywords: Homology modelling; Molecular dynamics; Bixin; Model validation

\section{Introduction}

Annatto (Bixa orellana L.) plant contains pigment contains bixin and norbixin, valuable natural colorants (Rodrigues et al.,2007). These pigments are widely used for industrial food and beverages, cosmetics, and as natural dyes for textiles (Chattopadhyay et al., 2008). Bixa orellana seed extracts also lowers blood glucose level by stimulating peripheral utilization of glucose so the seed might be of pharmacological importance (Russell et al., 2008). The natural dye bixin is extracted from the seed of the annatto plant and widely used as food colorant. Many restrictions are on the use of synthetic additives in the food industry as the synthetic dye having adverse effect but the investigation on biochemical properties of Bixa orellana plants and seed extracts has proven that it is having tumour inhibiting capacity (Reddy et al., 2005).Bixin is produced from the sub-pathway of isoprenoid biosynthesis. More specifically from the translycopene the bixin synthetic pathway diverges by the enzyme Lycopene cleavage oxygenase and further the action of intermediate enzymes like Lycopene beta cyclase and Lycopene epsilon cyclase lead to production of the pigment (Bouvier et al., 2003).So due to highly commercial and pharmaceutical importance and also to understand the biochemical and molecular basis of the biosynthesis of the natural compound is an interesting task. The first enzyme i.e. Lycopene cleavage oxygenase of the bixin synthesis pathway has been considered for the present study.The present study aim to model and validate the 3D structure of Lycopene cleavage oxygenase by computational approach whose structure has not been reported yet. Then to check the reliability of the model and validation purposes various tools and molecular dynamics method is used. This work may be further carried out for the biotechnology directed natural food colorant production in industrial level.

\section{Materials and Methods}

\section{Sequence retrieval and 3D model building}

The synthetic pathway of bixin production was obtained from the Metacyc pathway database, which is a sophisticated way to retrieve metabolic pathway data along with the sub biochemical reactions in detail (Caspi et al., 2010).The sequence for the Lycopene cleavage oxygenase enzyme was retrieved from Swissprot database from Expasy server (Schneider et al.,2004) and a protein BLAST search was performed against PDB (Protein Databank) to retrieve the corresponding template for the enzyme (Altschul et al.,1990). The model was built by MODELLER9v7 program and it uses an automated approach to comparative protein structure modelling (Eswar et al., 2008). In brief the modelling procedure begins with an alignment of the sequence to be modelled (target) with related known 3D structures (templates).The output is a 3D model for the target sequence containing all main chain and side chain non-hydrogen atoms (Andrej et al., 1993). The program also employs probability density functions (PDFs) derived analytically using statistical mechanics and empirically using a database of known protein structures as the spatial restraints.

\section{Model validation and Molecular dynamics study}

The MODELLER generated structure was verified for the missing side chains by SCWRL4 tool (Krivov et al.,2009) further verified by PROCHECK (Laskowski et al.,1993).The PROCHECK program provides the information about the stereo chemical quality of a given protein structure and was used to generate Ramachandrans plot. The DOPE (discrete optimized potential energy) score of modeller output per residues of the model was observed. DOPE score is calculated by Modeller program which is the distance dependent statistical potential based on probabilistic theory. This is extremely useful in making decisions about reliability (Shen et al., 2006).For the calculation of RMSD (Root mean square deviation) and Energy value of the model protein GROMACS 4.0.4 (Groingen Machine for Chemical Simulation) was used (Vander spoel et al., 2005).GROMACS is a versatile package to perform molecular dynamics that simulates

*Corresponding author: Raghunath Satpathy, Department of Biotechnology, MIRC LAB, MITS Engineering College, Rayagada, Odisha, India, Email: rnsatpathy@gmail.com

Received April 06, 2010; Accepted June 21, 2010; Published June 21, 2010

Citation: Satpathy R, Guru RK, Behera R, Priyadarshini A (2010) Homology Modelling of Lycopene Cleavage Oxygenase: The Key Enzyme of Bixin Production. J Comput Sci Syst Biol 3: 059-061. doi:10.4172/jcsb.1000057

Copyright: (c) 2010 Satpathy R, et al. This is an open-access article distributed under the terms of the Creative Commons Attribution License,which permits unrestricted use, distribution, and reproduction in any medium, provided the original author and source are credited. 
the Newtonian equations of motion for systems with hundreds to millions of particles (Hess et al., 2008). The model was subjected to molecular dynamics simulation in water at $300 \mathrm{~K}$ temperature and for 1000 pico second by using Gromos 43a1 force field of GROMACS tool. The computing facility utilised is High performance cluster for Biological Applications which is based on Intel Xeon Dual Quad core as processor, Gluster HPC 1.3 X86-64 bit edition ,total 16 nodes each having 4GB of memory.

\section{Results and Discussions}

\section{Homology modelling of Lycopene cleavage oxygenase}

The considered suitable template having PDB ID 2BIW (Kloer et al., 2005) for Lycopene cleavage oxygenase protein was obtained from BLAST search. Then Modeler9v7 program was used to generate the three dimensional structure (Figure 1). Modelling was performed for 10 times and the output of the model having lowest molpdf value was chosen as the final model.

\section{Structure validation by DOPE score and PROCHECK}

To verify further the predicted structures, The DOPE score for the model was obtained from Modeller output has been shown in (Figure 2). From the figure the peak indicates that there is no defect in the loop regions of the model. Also the validation of the model was carried out using Ramachandran plot calculations computed with the PROCHECK program. The $\Phi$ and $\Psi$ distributions of the Ramachandran plot of non-Glycine, non-Proline residues are summarized in (Figure 3 ). Altogether $100 \%$ of the residues were in favoured and allowed

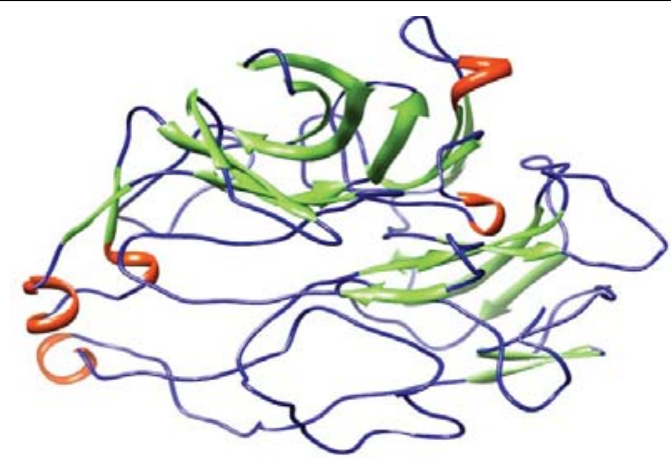

Figure 1: The final considered model from Modeller output (Visualisation by Chimera).

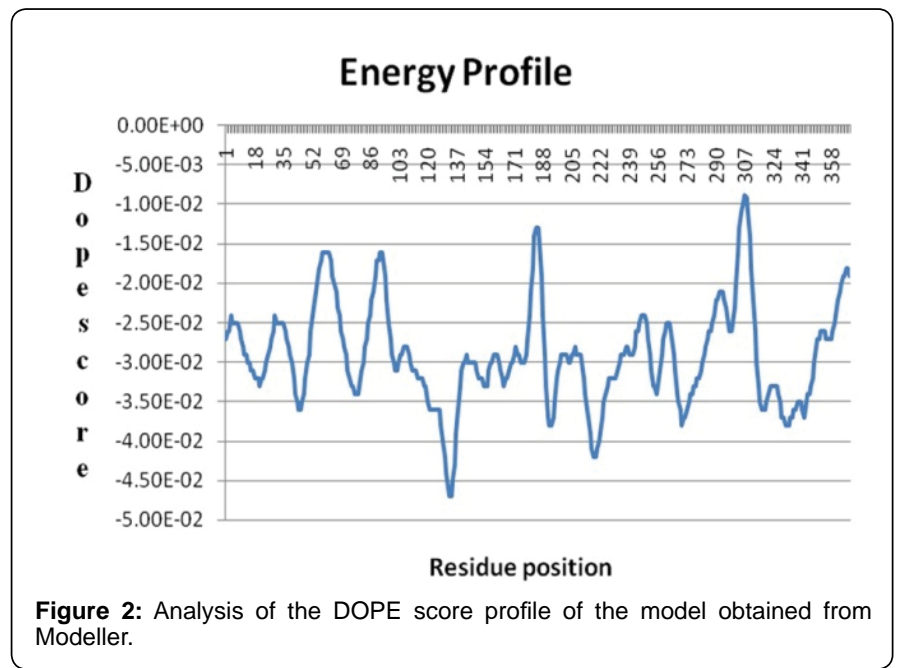

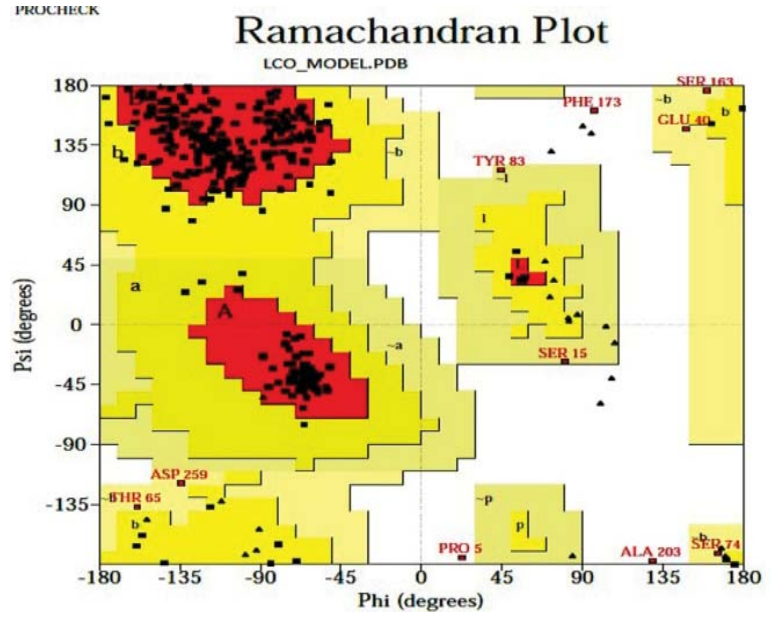

Figure 3: Ramachandran plot calculation of the psi/phi angle distribution of the model as computed by PROCHECK program.

\begin{tabular}{|l|l|}
\hline \% residues in favourable regions & 87.3 \\
\hline \% residues in additional residue regions & 9.7 \\
\hline$\%$ residues in generously regions & 2.3 \\
\hline$\%$ residues in disallowed regions & 0.6 \\
\hline \% of Non Proline and non Glycine residues & 100 \\
\hline Over all G- factor & -0.04 \\
\hline
\end{tabular}

Table 1: Ramachandrans plot calculations on 3D model of Lycopene cleavage oxygenase protein computed with the PROCHECK program.

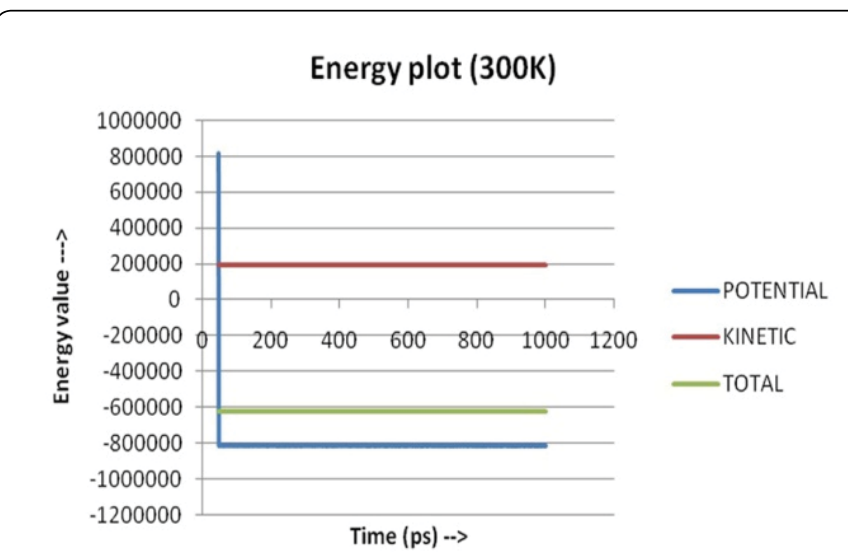

Figure 4: Energy plot obtained during 1000 Pico second molecular dynamics simulation of the model at $300 \mathrm{~K}$ temperature by Gromacs tool.

regions. The overall G-factor (Goodness factor) used was computed. (Table 1)

\section{Molecular dynamics simulation study}

The molecular dynamics simulation was performed. Both energy and root mean square deviation plots were derived from the respective trajectory file by Gromacs software output. The potential energy for the protein was initially high but during course of simulation it has been come down. Similarly the high kinetic energy shows the constant value however the total energy is low (Figure 4). The RMSD fluctuation plot shows the $C$-alpha backbone deviation during the simulation process is within the range 0.25-0.69 (Figure 5). Since it is a tolerable fluctuation in the backbone hence it confirms the model protein is stable.

Here in this work, we propose a valid and stable 3D model of 


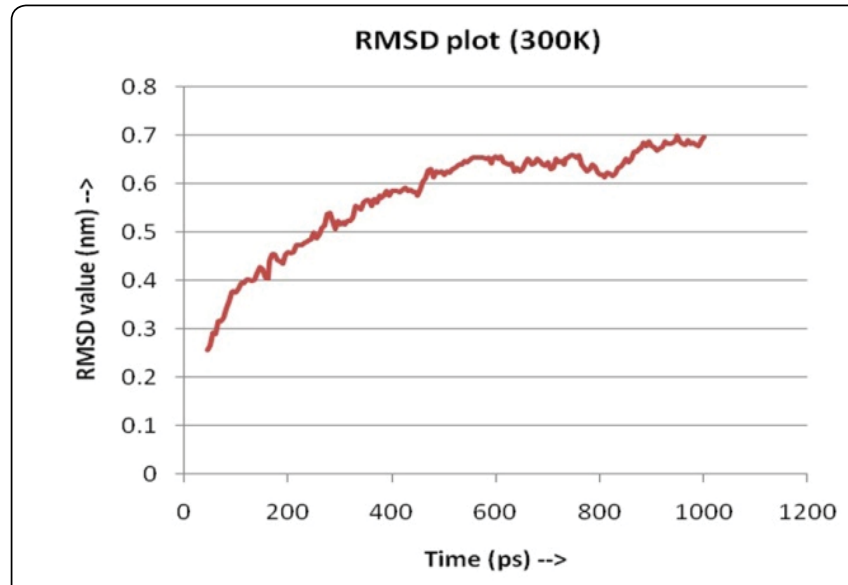

Figure 5: The Root mean square deviation plot (Carbon Alpha back bone) obtained from Gromacs tool during molecular dynamics simulation for 1000 Pico second.

Lycopene cleavage oxygenase enzyme whose structure is not present in PDB (Protein data bank) .Positive results obtained both from model validation followed by molecular dynamics simulation confirms our prediction. This in-silico work may be used further to predict the key residues in the substrate binding site of the enzyme that would lead to use of protein engineering approach to enhance the production of the important compound i.e. Bixin.

\section{Acknowledgement}

We are thankful to CEO, Director and Dean of Majhighariani Institute of Technology \& Science, Rayagada to provide us the MIRC lab for computing facility.

\section{References}

1. Altschul SF, Gish W, Miller W, Myers EW, Lipman DJ (1990) Basic local alignment search tool J Mol Biol 215: 403-410.

2. Bouvier F, Suire C, Mutterer J, Camara B (2003) Oxidative remodelling of chromoplast carotenoids: identification of the carotenoid dioxygenase CSCCD and CsZCD genes involved in Crocus secondary metabolite biogenesis Plant Cell 15: 47-62.
3. Chattopadhyay P, Chatterjee S, Sen SK (2008) Biotechnological potential of natural food grade biocolorants African Journal of Biotechnology 7: 2972 2985.

4. Caspi R, Altman T, Dale JM, Dreher K, Fulcher CA, et al. (2010) The MetaCyc database of metabolic pathways and enzymes and the BioCyc collection of pathway/genome databases. Nucleic Acids Res 38: 473-479.

5. Kloer DP, Ruch S, Al-Babili S,Beyer P,Schulz GE(2005) The structure of a retinal-forming carotenoid oxygenase. Science 308: 267.

6. Eswar N, Eramian D, Webb B, Shen MY, Sali A (2008) Protein structure modelling with MODELLER. Methods: Mol Biol 426: 145-159.

7. Hess B, Kutzner C, Van Der Spoel D, Lindahl E (2008) GROMACS 4: Algorithms for Highly Efficient, Load-Balanced, and Scalable Molecular Simulation J Chem Theory Comput 4: 435-447.

8. Krivov GG, Shapovalov MV, Dunbrack RL (2009) Improved prediction of protein side-chain conformations with SCWRL4 Proteins 77: 778-795.

9. Laskowski RA, MacArthur MW, Moss DS, Thornton JM (1993) PROCHECK: a program to check the stereo chemical quality of protein structures. J Appl Cryst 26: 283-291.

10. Reddy MK, Alexander-Lindo RL, Nair MG (2005) Relative inhibition of lipid peroxidation, cyclooxygenase enzymes, and human tumor cell proliferation by natural food colors. J Agric Food Chem 53: 9268-9273.

11. Russell KR, Omoruyi FO, Pascoe KO, Morrison EY (2008) Hypoglycaemic activity of Bixa orellana extract in the dog Methods Find. Exp Clin Pharmacol 30: 301-305.

12. Rodrigues SM, Virgínia LF Soares, De Oliveira TM, Gesteira AS, Otoni WC et al. (2007) Isolation and purification of RNA from tissues rich in polyphenols, polysaccharides, and pigments of annatto (Bixa orellana L). Mol Biotechnology 37: $220-224$.

13. Shen MY, Sali A (2006) Statistical potential for assessment and prediction of Protein structures. Protein Sci 15: 2507-2524.

14. Sali A, Blundell TL (1993) Comparative protein modelling by satisfaction of spatial restraints. Mol Biol 234: 779-815.

15. Schneider M, Tognolli M, Bairoch A (2004) The Swiss-Prot protein knowledgebase and ExPASy: providing the plant community with high quality proteomic data and tools. Plant Physiol and Biochem 42: 1013-1021.

16. VanDer Spoel D, Lindahl E, Hess B, Groenhof G, Mark AE, et al. (2005) GROMACS: Fast, Flexible and Free. J comp chem 26: 1701-1718. 